\title{
Focus on psychiatry in Singapore
}

\author{
EE HEOK KUA
}

This is an overview of the historical development of psychiatric services and the progress of psychiatric research and training in Singapore. With a population of 4 million, the city is evolving from developing to developed status and the concomitant social implications pose enormous challenges for mental health professionals. The demographic changes in recent years, with an ageing population and with more women (the traditional family caregivers) in the workforce, have had profound consequences for the delivery of health care services. In this cosmopolitan city of Chinese, Indians and Malays, myriad cultures and beliefs influence the health-seeking behaviour and the care of people with mental illness.

\section{HISTORICAL BACKGROUND}

Soon after the establishment of a British trading post on the island of Singapore in 1819, an assistant surgeon from the East India Company was responsible for the provision of medical care to the settlers and troops. People with mental illness were initially incarcerated in the local jail until a 30-bed 'insane hospital' was built in 1841. With the growth of the port and increase in the population, it was decided by the Colonial Office in London to build a larger asylum. Woodbridge Hospital, with a capacity of more than 2000 beds, was opened in 1928 and has remained the principal psychiatric institution on the island. The British Governor had also stipulated that the Medical Superintendent appointed to the Hospital should be an officer 'with some taste for gardening and farming, who will help to make the patients interested in such pursuits. Must have attended lectures at the Mental Hospital, Denmark Hill. Must possess the Diploma in Psychological Medicine'. The decree of the Maudsley connection is heeded even to this day- every local Medical Superintendent or Director has had training at the Maudsley and possesses the DPM or MRCPsych; but horticultural predilection is no longer a requirement.

Like most psychiatric institutions built in that period, Woodbridge Hospital, fringed by the jungle and rubber plantations, was sequestered at a corner of the island. In the 1960 s community psychiatry was in its infancy and psychiatric clinics were started in a few government outpatient departments in the city. The problems of opium addiction and delinquency were dealt with mainly by social workers, who also managed psychiatric referrals in the general hospitals (e.g. for attempted suicide). In the 1970 s with only a few trained psychiatrists, other mental health professionals - psychologists, nurses and social workers - were recruited. An Association for Mental Health was formed as a non-governmental organisation to promote mental health and provide day care facilities for discharged patients.

The 1980s saw accelerated progress in psychiatric education with the establishment of the Department of Psychological Medicine at the National University of Singapore to teach medical students and to coordinate psychiatric research. The Department was first based at the Singapore General Hospital and moved to the new National University Hospital in 1987. During this period more day care centres were set up for psychiatric patients, children with learning disabilities and the frail elderly. Most of these services were staffed by nurses, psychologists or social workers. By the end of the 1990s, there were psychiatric out-patient clinics in all the general hospitals and major out-patient departments.

Because of the stigma of mental illness, the well-heeled prefer to seek psychiatric treatment in private clinics. Of the 120 psychiatrists in the island, about one-fifth are in private practice.

\section{TRADITIONAL MEDICINE}

In Asian societies, indigenous theories of health and illness are embedded in customs and religious philosophies. Family structures and cultural beliefs often determine illness behaviour and help-seeking tendencies. In a study of illness behaviour in 100 Chinese patients referred consecutively to the psychiatric clinic at the National University Hospital, it was found that 36 had also consulted a traditional healer (Kua et al, 1993). More women than men felt that their illness was due to spirit possession; but belief in possession was not related to educational status.

Classical Chinese medicine is based on the belief that there is a finely balanced and rhythmic relationship between bodily functions and the emotions. This belief is built on the concept of yin-yang, a bipolarity that is both opposite and complementary. The yin represents coldness and yang warmth. When this homoeostasis is disrupted by spirits, mental illness might result. In the family it is often the elders who seek help from the traditional healer to intercede for the patient to exorcise the spirits. If the traditional healer fails, the family might then consult a general practitioner who will refer the patient on to the psychiatrist if he has difficulty with his or her management.

The possession-trance is a common culture-related phenomenon in Singapore and many countries in Asia. I have previously reported the characteristic features (Kua, 1986). People prone to this condition are often from less-advantaged backgrounds and have received poor education, they have previously witnessed a trance and the onset is usually before the age of 25 years. During the trance there is evidence of an alteration in the level of consciousness, and stereotyped behaviour of a deity which has possessed the person. The trance lasts for less than an hour, and is followed by physical exhaustion and amnesia for the period of the trance, with normal behaviour in the interval between trances. The young men in the study had these experiences soon after enlistment in the army, which was perceived as a stressful life event. Because possession-trance is not deemed an illness, a traditional healer is often consulted. This socially sanctioned behaviour is recognised as a sign of distress and evokes the appropriate family response of support and sympathy. The individual is treated with respect because he is perceived 
to be favoured by a deity. The healer shares the same belief system as the family, whose trust and hope are powerful factors in the treatment. During the therapy, the healer goes into a trance himself and the family participates in the rituals. This phenomenon can be explained as a defence mechanism to preserve self-dignity and self-worth. Treatment by the traditional healer lacks the stigma associated with referral to a psychiatric hospital.

The Malays and Indians have their own priests or healers who are consulted not only for spiritual matters, but also when someone is ill. Among the different ethnic groups, there are other culture-related conditions such as amok and koro which are less common now. One of the earliest reports on amok was written in 1893 by the British psychiatrist in Singapore, Dr W. G. Ellis. He concluded that amok was a homicidal-suicidal rage due to depression. The patient would often describe his mood state in the vernacular as sakit hati or sickness of the liver, the organ regarded as the seat of the emotions (Kua, 1991).

Because of their cultural beliefs, patients who seek psychiatric treatment will take medication from their doctor and also a herbal prescription from a traditional healer. Sometimes patients with schizophrenia who seek help from traditional healers are referred for psychiatric treatment after a delay of 2-3 years. With public education, we have noticed recently that the majority of patients with first-episode schizophrenia $(80 \%)$ are referred to the National University Hospital within 6 months of the illness onset.

\section{PSYCHIATRIC SERVICES}

There are public and private health care systems to provide primary and secondary care. The public hospitals have been restructured in recent years to allow some autonomy in governance. In general, doctors in primary care do not manage psychiatric patients except for those with mild non-psychotic disorders. Because of stigma and the desire for confidentiality, some patients prefer to use private psychiatric services. Besides Woodbridge Hospital, there are in-patient services in all the major general hospitals. As in most countries, the profile of psychiatric patients in general hospitals differs from that of those in psychiatric hospitals with a greater proportion of patients with psychotic disorders in the latter.
Sub-specialties such as forensic psychiatry, substance misuse and psychiatric rehabilitation are mainly based in the psychiatric hospital. The Child Guidance Clinic has been well established for the past 20 years. With an ageing population, there is a growing interest in old age psychiatry. More mental health professionals have now been trained in psychotherapy with the introduction of the Diploma of Psychotherapy by the National University of Singapore 4 years ago.

As in most developing countries, there is an acute shortage of mental health professionals. There is no luxury of time and it is not uncommon in a clinic to see between 10 and 20 patients in 3 hours. To help with the quick assessment of elderly patients with cognitive impairment we have constructed a 10-item questionnaire called the Elderly Cognitive Assessment Questionnaire (ECAQ) that can be used for screening for dementia (Kua \& Ko, 1992a). The Mini-Mental State Examination (MMSE) was used previously but it was extremely difficult to administer because of cultural differences, linguistic problems and the low literacy rate in our elderly population. The ECAQ has less cultural and educational bias and is now used by doctors and nurses in many Asian countries.

After discharge from hospital, the majority of patients return to their families. The social transformation of the Singaporean family has affected the care of patients because there are fewer family caregivers as a result of the low birth rate since the 1970s and more women in the workforce. The stress burden of caregivers of patients with dementia has been highlighted (e.g. Kua \& Tan, 1997). The need to expand and improve community psychiatric services to support families has been raised in Parliament.

\section{UNDERGRADUATE TEACHING}

The teaching objective of the undergraduate curriculum is to provide medical students with a good foundation in psychiatry necessary for primary care practice. There is an emphasis on history-taking, mental state examination and communication skills. During the 6-week clerkship (4 weeks in the third year and 2 weeks in the final year) the students are exposed to a wide range of psychiatric disorders, with an emphasis on the common problems of anxiety and depression. Besides pharmacotherapy, they are also taught techniques of counselling and stress management.

The integrated curriculum of the medical school encourages combined teaching sessions with related disciplines such as neuroanatomy, neurophysiology, psychopharmacology, psychology, neurochemistry and neurology.

Medical students in their first and second years are expected to conduct a small research project on any topic related to medicine. Psychiatry is a popular discipline and some of the projects submitted include: reminiscence therapy for the elderly, sleep problems of junior doctors, eating disorders, the cost of health care in dementia, and rehabilitation in drug addiction.

Most textbooks of psychiatry for medical students are from the UK or USA. Because of the differences in clinical presentation, availability of services and legal systems, we have pooled our collective experiences to publish a textbook for our students and general practitioners. This textbook, Psychiatry for Doctors (Kua et al, 1995), is also used by doctors in many other Asian countries.

It is important to view the medical students' posting as an opportune moment to demystify mental illness and to present a more positive image of psychiatry. It is paradoxical that the problem of stigmatisation is sometimes at its worst among our medical colleagues. The students who have a good impression of the posting will convey this positive image to their friends and families.

\section{POSTGRADUATE TRAINING}

Before 1987 doctors wanting to specialise in psychiatry were sent on scholarships to the Institute of Psychiatry in London. As postgraduate medical education in Singapore expanded in the 1980 s, the need for a local Master of Medicine (Psychiatry) was mooted. There is a compelling reason to build the basic foundation from local training before venturing overseas for further training. Moreover, besides the cultural and linguistic issues, psychiatric practice in London is very different from that in Singapore. The University sought advice from the Royal College of Psychiatrists and Professor Philip Seager visited Singapore to help in planning the training programme. There are some similarities with the British model - a 3-year basic specialist 
training with a two-part examination before moving on to advanced traineeship. During the next 3 years the trainee must submit a research project at the exit examination before certification as a specialist in psychiatry. The external examiners are mainly from the UK and Australia.

Senior registrars are encouraged to subspecialise and they are sent for training overseas. In the past, most psychiatrists would make a beeline for the Maudsley, but recently we have sent them to other centres of excellence in the UK or elsewhere in Europe, the USA or Australia.

After a prolonged gestation phase, the Graduate Diploma of Psychotherapy conducted by the Faculty of Medicine started 4 years ago. We had the assistance of Drs Roy Menninger, Christopher Dare and Jeremy Holmes to train the supervisors and plan the course. The 1-year programme is in dynamic psychotherapy and cognitivebehavioural psychiatry.

\section{RESEARCH}

Psychiatric research has focused on a few key areas, including schizophrenia, suicide, dementia and addiction. Although publications are predominantly on clinical research and epidemiology, there is a growing interest in basic sciences and genetics.

The Department of Psychological Medicine is one of the research centres for dementia in Asia. We have published papers on the epidemiology of dementia and depression in Chinese and Malay elderly using the Geriatric Mental State schedule (Kua, 1992; Kua \& Ko, 1995). The data showed that the prevalence of Alzheimer's disease was quite similar for elderly Chinese and Malays but the prevalence of vascular dementia was highest among elderly Malay women. A public health survey also indicated that Malay women were more prone to diabetes mellitus, hypertension and obesity. This has been highlighted to the Malay community in public education and we hope that preventive measures will help to lower the prevalence of vascular dementia.

The high suicide rate in the elderly (Kua $\&$ Ko, 1992b) prompted us to focus on depression in the elderly. In a community study we found that about $40 \%$ of elderly people with depression had comorbid anxiety disorders (Kua et al, 1996) and the 5year outcome of depression was bleak (Kua, 1993). There are plans to embark on a study of risk factors in depression so that we can consider preventive strategies and early intervention.

A National Mental Health Survey was conducted in 1996 using a two-phase design - initial screening with the General Health Questionnaire, followed by structured interview of probable cases with the Composite International Diagnostic Interview (Fones et al, 1998). Using cut-offs validated for each ethnic group, the minor psychiatric morbidity rate for Chinese was $17.4 \%$, Malays $15.1 \%$ and Indians $17.8 \%$. The ICD-10 psychiatric disorders were mainly anxiety and depression. The study also indicated a gender difference with a higher prevalence in working women.

The long-term outcome of schizophrenia is well studied because it is not too difficult to trace patients living in a small island and patients' records are usually well kept. In a follow-up study of patients with schizophrenia admitted to Woodbridge Hospital in 1975, Tsoi \& Kua (1992) found that after 10 years, $29 \%$ were well and working, $24 \%$ were well but not working, $24 \%$ were unwell and on treatment, and the remaining $23 \%$ required long-term care. A paper on the 20-year follow-up of this cohort will be published soon. Recently, the thrust of research has been on the prodromal symptoms of schizophrenia (Tan \& Ang, 2001) with the hope that early intervention will prevent chronicity.

The cosmopolitan communities have wide differences in psychiatric disorders. The ethnic variation in elderly suicide indicated a high rate for Chinese and a very low rate for Malays (Kua \& Ko, 1992b; Kua et al, 2003). A publication on suicide following parasuicide showed that the rate after 3 years was $0.67 \%$, which was much lower than in Britain (Tsoi \& Kua, 1987). There are ethnic differences in addictive behaviour - heroin addiction is more common in Malay youth, alcohol misuse among the Indians and gambling among the Chinese. The changing lifestyle of young people has seen a rise in alcohol consumption, especially among the Chinese, and referrals for treatment have increased (Kua et al, 1990; Kua, 1995).

\section{GOVERNMENTAL AND NON-GOVERNMENTAL ORGANISATIONS}

It is undeniable that governmental support is paramount for the success of any mental health programme. Government policies often reflect the needs of the electorate. The health authorities seek the opinion of specialists if there is public concern about issues such as the suicide rate, stress in family caregivers, and addiction. In our dialogue with government officials, we find that it is always advantageous to be armed with data from local research to help them understand the national issues. For example, from a study on dementia (Kua, 1992), we demonstrated that a prevalence of $2.5 \%$ would equate to an absolute number of about 6000 cases in Singapore today and 12500 in the year 2030. There is, therefore, the need for more community services for the elderly in future.

Although the suicide rate in the elderly was at a peak in 1996, the combined effort of the government, non-governmental organisations and mental health professionals has resulted in a gradual decline in the past few years (Kua et al, 2003).

We have convinced the health authorities to fund new community programmes for addiction treatment and the early management of psychosis.

The pivotal role of non-governmental organisations, especially religious groups, in psychiatric service delivery is evident in their many community facilities for drug rehabilitation, dementia care and the long-term care of patients with chronic schizophrenia.

\section{DE-STIGMATISATION CAMPAIGN}

In the past 10 years there has been a concerted effort by mental health professionals to campaign against the stigma of mental illness. We have funding from the government for public education targeting children, working people and retired people. Some of this takes the form of talks given in schools, hotels, community centres and workplaces to promote mental health and to educate the public about mental illness.

A study on stigma has highlighted the distress of patients and staff (Lai et al, 2000). The results of research on homicide and mental illness (Kua et al, 1985) have often been quoted to debunk the myth of the dangerous psychiatric patient.

The anti-stigma campaign 'Changing Hearts and Minds' has the cooperation of the mass media and there are regular columns in the daily papers and air time on 
radio and television for mental health programmes.

\section{THE ROYAL COLLEGE OF PSYCHIATRISTS AND THE OVERSEAS MEMBERS}

Besides the monthly Journal and yearly reminder about the membership fee, overseas members do not have any links with the College. From this distant corner of the globe, the affairs of the College seem unimportant and irrelevant. To strengthen relationships the College could hold training workshops, regional conferences or even conduct the MRCPsych examinations in Singapore. It would also be an opportunity for Western doctors to explore the East. (After I had presented a paper at a meeting in London 10 years ago, one symposiast asked me 'Whereabouts in China is Singapore?')

\section{FUTURE PROGRAMMES}

With the capricious economic climate in Asia, cost containment is now the political catchword. Health economics has become a topical issue in hospital board meetings. Workforce and funding issues are crucial for any development or expansion of psychiatric services. In spite of these uncertainties we hope to continue our training and research programmes with the collaboration of other Asian countries.

There is tacit agreement among senior psychiatrists in the Asian region to have regular consultation to coordinate training and research. We had a meeting in Kobe University 2 years ago with Professors Norman Sartorius and Naotaka Shinfuku about studying the prescribing pattern of antipsychotic medication. This project involved the participation of Japan, China, South Korea, Taiwan, Hong Kong, Malaysia, Indonesia and Singapore and the results were presented at the World Congress of the World Psychiatric Association at Yokohama in 2002.

An Asian regional teachers of psychiatry meeting has been planned this year with the involvement of Australian colleagues, including Professor Edmond Chiu. It will be a workshop to train the trainers. We look forward to the participation of the College in future meetings of this kind.

\section{CONCLUSION}

The past two decades have witnessed a gradual transformation of psychiatric services from institutional to community care. Ultimately we hope to provide a service that is acceptable and comprehensive. Health costs are escalating and hospitalisation is expensive. The families of psychiatric patients will need more community psychiatric services. In tandem with the changes, there has been progress in psychiatric training and research.

After reading this article I hope you have learned something about the challenges of psychiatry in Singapore. If you have not, you should at least search an atlas and learn that Singapore is not in China but is a small island at the south-eastern tip of the Asian land mass in the midst of the Malay archipelago.

\section{REFERENCES}

Fones, S. C. L., Kua, E. H., Ko, S. M., et al (1998) Studying the mental health of a nation. Singapore Medical Journal, 39, 25I-255.

Kua, E. H. (1986) A cross-cultural study of the possession-trance in Singapore. Australian and New Zealand Journal of Psychiatry, 20, 36I-364.

Kua, E. H. (1987) Drinking in Chinese culture: old stereotypes re-examined. British Journal of Addiction, 82, 224-225.

Kua, E. H. (1991) Amok in nineteenth-century British Malaya history. History of Psychiatry, 3, 429-436.

Kua, E. H. (1992) A community study of mental disorders in elderly Singaporean Chinese using the GMS-AGECAT package. Australian and New Zealand Journal of Psychiatry, 26, 502-506.
Kua, E. H. (1993) The depressed elderly Chinese in the community: a five year follow-up study. International Journal of Geriatric Psychiatry, 8, 427-430.

Kua, E. H. (1995) A profile of Chinese alcoholics. Addiction, 90, 5I-56.

Kua, E. H. \& Ko, S. M. (1992a) A questionnaire to screen for cognitive impairment among elderly people in developing countries. Acta Psychiatrica Scandinavica, 85, 119-122.

Kua, E. H. \& Ko, S. M. (1992b) A cross-cultural study of suicide among the elderly in Singapore. British Journal of Psychiatry, 160, 558-559.

Kua, E. H. \& Ko, S. M. (1995) Prevalence of dementia among elderly Chinese and Malay residents of Singapore. International Psychogeriatrics, 7, 439-446.

Kua, E. H. \& Tan, S. L. (1997) Stress of caregivers of dementia patients in the Chinese family. International Journal of Geriatric Psychiatry, 12, 466-469.

Kua, E. H., Yuan, A. F. \& Ang, A. L. (1985) Homicide in Singapore. Medicine, Science and the Law, 25, 26-28.

Kua, E. H., Ko, S. M., Tian, C. S., et al (1990) A followup study of Asian problem drinkers. British Journal of Addiction, 85, 1261-1264.

Kua, E. H., Chew, P. H. \& Ko, S. M. (1993) Spirit possession and healing among Chinese psychiatric patients. Acta Psychiatrica Scandinavica, 88, 447-450.

Kua, E. H., Ko, S. M. \& Lim, C. C. (1995) Psychiatry for Doctors. Singapore: Armour Academics.

Kua, E. H., Ko, S. M., Fones, C. S. L., et al (1996) Comorbidity of depression in the elderly. International Journal of Geriatric Psychiatry, II, 699-704.

Kua, E. H., Ko, S. M. \& Ng, T. P. (2003) Recent trend in elderly suicide rates in a multiethnic Asian city. International Journal of Geriatric Psychiatry, 18 533-536.

Lai, Y. M., Hong, C. \& Chee, C. (2000) Stigma of mental illness. Singapore Medical Journal, 42, III-114.

Tan, H. Y. \& Ang, Y. G. (200I) First episode psychosis in the military - a comparative study of prodromal symptoms. Australian and New Zealand Journal of Psychiatry, 35, 512-519.

Tsoi, W. F. \& Kua, E. H. (1987) Suicide following parasuicide in Singapore. British Journal of Psychiatry, I5I, 543-545.

Tsoi, W. F. \& Kua, E. H. (1992) Predicting the outcome of schizophrenia ten years later. Australian and $\mathrm{New}$ Zealand Journal of Psychiatry, 26, 257-26l.

Ee Heok Kua Department of Psychological Medicine, National University Hospital, 5 Lower Kent Ridge Road, Singapore 119074.

E-mail: pcmkeh@nus.edu.sg 